\section{Evaluación de la anticoagulación con rivaroxaban, en pacientes con fibrilación auricular no valvular de reciente diagnóstico}

\author{
VÍCTOR NEIRA ${ }^{1}$, RAMÓN CORBALÁN ${ }^{1}$, JAIME PEREIRA ${ }^{2}$, \\ OLGA PANES $^{2, a}$, BERNARDITA GARAYAR ${ }^{1}$, ANDRÉS AIZMAN $^{1}$, \\ SILVANA LLEVANERAS ${ }^{1, b}$, LUIS VILLARROEL ${ }^{3, c}$
}

\section{Evaluation of oral anticoagulation with rivaroxaban, in patients with new onset non valvular atrial fibrillation}

Background: Atrial fibrillation (AF) generates a hypercoagulable state with an increased thrombin generation and raised levels of thrombin-antithrombin complexes, which results in a high risk of stroke and thromboembolism. Aim: To evaluate the anticoagulant effect of rivaroxaban by anti-Xa factor activity and its correlation with thrombin-antithrombin complexes, thrombin generation and prothrombin time in patients newly diagnosed with non-valvular AF. Patients and Methods: Prospective study in patients with indication of anticoagulation. Demographic variables, cardiovascular risk factors, $C H A_{2} D S_{2}-V A S c$ and HAS$B L E D$ scores were recorded. Blood samples were taken at baseline, at 3 and 24 hours after the administration of the drug and at 30 days. Rivaroxaban levels, anti-Xa activity, prothrombin time, thrombin generation and plasma levels of thrombin-antithrombin complexes were determined. Results: We studied 20 patients aged $76.3 \pm 8.0$ years ( $60 \%$ female) with a $\mathrm{CHA}_{2} D S_{2}$-VASc score $>2$ points. The anti-Xa factor activity correlated with rivaroxaban plasma levels at 3 hours $(r=0.61, p<0.01)$, at 24 hours $(r=0.85, p<0.01)$ and at 30 days $(r=0.99, p<0.01)$, with prothrombin time at 3 hours $(r=-0.86, p=0.019)$ and at 30 days $(r=-0.63, p=0.02)$ and with a sustained decrease in thrombin generation at 30 days of follow-up $(r=-0.74, p<0.01)$. There was no correlation with thrombin-antithrombin complexes $(r=-0.02, p=0.83)$. Conclusions: $R i$ varoxaban consistently inhibited the mild pro-coagulant state found in newly diagnosed non-valvular AF patients through the first 24 hours and this effect was maintained at 30 days. Plasma levels of the drug correlated with anti-Xa factor activity, thrombin generation and prothrombin time

(Rev Med Chile 2016; 144: 1103-1111)

Key words: Atrial Fibrillation; Rivaroxaban; Thrombin; Thrombin Time.

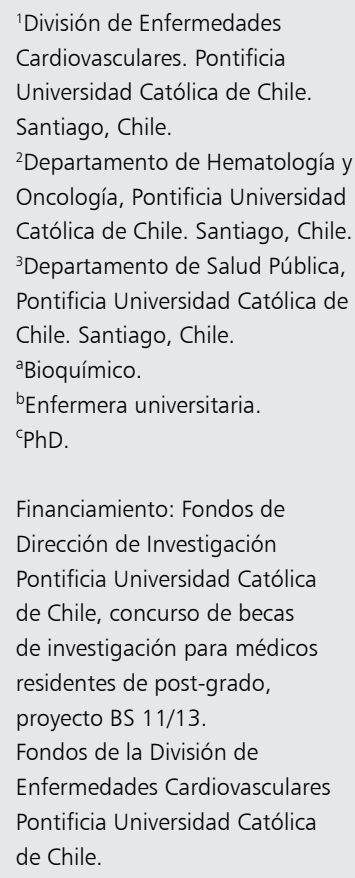

Financiamiento: Fondos de Dirección de Investigación Pontificia Universidad Católica de Chile, concurso de becas de investigación para médicos residentes de post-grado, proyecto BS 11/13.

Fondos de la División de Enfermedades Cardiovasculares Pontificia Universidad Católica de Chile.

Recibido el 15 de marzo de 2016 aceptado el 12 de julio de 2016.

Correspondencia a:

Dr. Víctor M. Neira Vidal Hospital Clínico Pontificia Universidad Católica de Chile. Marcoleta 367. División de Enfermedades Cardiovasculares, Santiago, Chile.

código postal 8330024

Teléfono + 56-2-3543633

victorneirav@gmail.com
L a introducción de nuevos anticoagulantes orales directos ha significado un progreso en la prevención del tromboembolismo sistémico en pacientes con fibrilación auricular (FA) no valvular. Una dosificación simple, estabilidad de su efecto anticoagulante, menor interacción con drogas y alimentos, unido a un perfil de seguridad comparable o superior al de antagonistas de la vitamina $\mathrm{K}$ constituyen propiedades atractivas para su empleo en clínica y su utilización ha ido en un constante aumento en el mundo ${ }^{1-7}$.

Una de las limitaciones importantes para 
su empleo ha sido la falta de un método que permita establecer el grado de inhibición de la coagulación en un momento determinado, el que tradicionalmente se ha medido a través del tiempo de protrombina estandarizado por el INR, cuando se prescriben antagonistas de la vitamina K. Esta dificultad se acentúa para muchos clínicos cuando se les recomienda el empleo de nuevos anticoagulantes en dosis única diaria o en dosis divididas cada $12 \mathrm{~h}^{8}$.

En el caso de rivaroxaban, un inhibidor del factor $\mathrm{Xa}(\mathrm{FXa})$, hay estudios in vitro, en voluntarios sanos y en pacientes con trombosis venosa que demuestran una relación estrecha de los niveles plasmáticos de la droga con la inhibición del $\mathrm{FXa}^{9-10}$. Otros estudios demuestran una reducción transitoria del tiempo de protrombina en el momento del efecto máximo de la droga y también un retardo de la generación de trombina ${ }^{11}$.

En el presente estudio hemos evaluado el efecto inicial y sostenido de rivaroxaban en una dosis única sobre distintos marcadores de la coagulación sistémica, en un grupo de pacientes con FA no valvular de riesgo moderado.

\section{Material y Método}

Estudio prospectivo en el que se reclutaron pacientes evaluados en forma ambulatoria o en el Servicio de Urgencia de la Red de Salud del Hospital Clínico de la Pontificia Universidad Católica de Chile, con FA no valvular de reciente diagnóstico e indicación de inicio de tratamiento anticoagulante oral (puntaje $\mathrm{CHA}_{2} \mathrm{DS}_{2}$-VASc $\geq 2$ puntos).

Los pacientes invitados a participar en el estudio firmaron en forma previa un consentimiento informado aprobado por Comité de Ética en Investigación, de la Escuela de Medicina de la Pontificia Universidad Católica de Chile.

Fueron criterios de inclusión pacientes mayores de 18 años con diagnóstico reciente de FA no valvular de menos de 6 semanas de evolución y con indicación de inicio de terapia anticoagulante oral según puntaje de riesgo.

Se excluyeron pacientes con síndrome coronario agudo o cirugía cardiaca recientes ( $<6$ meses), historia o hallazgo de valvulopatías, enfermedades infecciosas, inflamatorias, neoplásicas o afecciones tiroídeas en evolución.

Se registraron variables demográficas, factores de riesgo cardiovascular, función ventricular y uso de fármacos de acción cardiovascular. En todos los pacientes se efectuó un ecocardiograma para medir diámetro y volumen de aurícula izquierda, diámetros de ventrículo izquierdo, evaluación de motilidad segmentaria, grosor de sus paredes y determinación de fracción de eyección.

Se tomaron muestras de sangre previo al inicio de tratamiento anticoagulante con rivaroxaban y luego otras a las 3 y $24 \mathrm{~h}$ posterior a la primera dosis. A los 30 días de tratamiento se tomaron nuevas muestras, $24 \mathrm{~h}$ después de la última dosis.

Para los propósitos de esta investigación, se recomendó a los pacientes tomar rivaroxaban junto con el desayuno; la dosis recomendada para la prevención de accidente cerebro vascular en fibrilación auricular no valvular es de $20 \mathrm{mg}$ diarios y se ajustó la dosis a $15 \mathrm{mg}$ diarios, en caso de insuficiencia renal moderada, con clearance de creatinina entre $50-30 \mathrm{~mL} / \mathrm{min}$. La enfermera a cargo del protocolo de seguimiento se encargó de controlar la administración adecuada del fármaco y contactar a los pacientes para la toma de muestra.

Se determinaron los niveles plasmáticos de rivaroxaban mediante su actividad anti-FXa mediante un ensayo cromogénico $\left(\mathrm{STA}^{\circledR}\right.$ liquid anti-Xa, Diagnostica Stago) en coagulómetro ACL $\mathrm{TOP}^{\circledR}$ Analyzer (Instrumentation Laboratory, Bedford MA, USA) ${ }^{12}$. Se utilizaron calibradores específicos para rivaroxaban cubriendo un rango de $0-472 \mathrm{ng} / \mathrm{mL}$, con una sensibilidad de $25 \mathrm{ng} /$ $\mathrm{mL}$ según especificaciones del fabricante.

Se determinaron los siguientes factores de la coagulación: a) tiempo de protrombina (TP), por técnica estándar usando HemosIL ${ }^{\circledR}$ Recombi Plas Tin en coagulómetro ACL TOP; b) actividad anti-FXa con la misma técnica, pero con el uso de calibradores para heparina no fraccionada y heparina de bajo peso molecular, con límite de detección de $0,1 \mathrm{U} / \mathrm{mL}$; c) niveles plasmáticos de complejos TAT por técnica de Elisa (Affinity Biological); d) capacidad de generación de trombina (GT), que se evaluó en plasma pobre en plaquetas y plasma rico en plaquetas. Para la obtención de plasma pobre en plaquetas, se procesó sangre anticoagulada con citrato de sodio a $3,2 \%$, la cual fue centrifugada a $3.000 \mathrm{~g}$ por $10 \mathrm{~min}$ y luego a 10.000 g por $7 \mathrm{~min}$. La GT en plasma pobre en plaquetas fue realizada como ha sido previamente descrita ${ }^{13}$.

Brevemente, $80 \mu \mathrm{l}$ de plama pobre en plaquetas se mezcla con buffer conteniendo $5 \mathrm{pM}$ de factor 
tisular recombinante y $0,8 \mu \mathrm{M}$ de vesículas de fosfolípidos. La reacción se inició por adición de sustrato y $0,1 \mathrm{M}$ de $\mathrm{CaCl}$. El área bajo la curva, el pico máximo y la velocidad de generación de la trombina se calculó usando un software dedicado. Para determinar la GT en plasma rico en plaquetas, se utilizó sangre anticoagulada con citrato, se centrifugó a 240 g por 10 min y el sobrenadante (plasma rico en plaquetas) fue activado en agregómetro con una mezcla de factor von Willebrand y ristocetina. Posteriormente, $100 \mu \mathrm{L}$ de plasma rico en plaquetas activado se usó para determinar la GT en las mismas condiciones y equipo que para plasma pobre en plaquetas, pero sin la adición de factor tisular ni fosfolípidos ${ }^{14}$.

\section{Cálculo de tamaño muestral}

Se calculó una muestra de 16 sujetos para encontrar estadísticamente significativa una correlación mayor o igual a 0,6 de la actividad anti-FXa y los niveles plasmáticos de complejo TAT y GT plasmática, con una potencia de $80 \%$ y con un nivel de confianza de $95 \%$. Se incluyeron 4 sujetos más ante la eventualidad de pérdida de seguimiento.

\section{Análisis estadístico}

Se describieron los sujetos en cuanto a características demográficas, prevalencia de factores de riesgo cardiovascular, función ventricular. Se realizó un análisis exploratorio de los datos, identificando aquellos valores fuera de rango, datos faltantes, datos duplicados y errores de digitación. En las variables continuas se estudió su distribución con el fin de determinar el promedio y su desviación estándar o la mediana y percentiles 25-75 para su descripción, dependiendo de su normalidad. Para los propósitos de estratificación de riesgo tromboembólico se utilizó el puntaje de riesgo $\mathrm{CHADS}_{2} \mathrm{VASc}_{2}$. Se utilizó la prueba de " $\mathrm{t}$ ", test para muestras pareadas cuando los parámetros de una distribución sean normales, y la prueba no paramétrica (test de rangos signados de Wilcoxon) en caso contrario. Se utilizó la prueba exacta de Fisher para medidas categóricas.

Se realizó un análisis pareado sobre el efecto del rivaroxaban en la inhibición del FXa y su relación con los niveles plasmáticos de complejo TAT, GT y TP, en diferentes tiempos, en muestras repetidas, explorando su correlación. Se realizó análisis de las diferentes fases de tomas de muestra (basal, a las 3 y 24 h posterior a la primera dosis de rivaroxaban y a los 30 días de tratamiento) con el test de ANOVA para medidas repetidas y test de comparaciones múltiples de Hochberg para detectar el o los promedios que son distintos según el test de ANOVA. La validación de las bases de datos y los análisis estadísticos fueron realizados mediante el programa SAS/STAT software ${ }^{\circledR}$.

\section{Resultados}

Se enrolaron 20 pacientes durante el período agosto de 2013-junio de 2014, a todos los cuales se les realizó seguimiento prospectivo. Las características basales de los sujetos en estudio se describen en la Tabla 1. De ellos, $60 \%$ corresponde a sexo femenino, la edad promedio fue de 76,3 $\pm 8,0$ años, $85 \%$ presentó fibrilación auricular paroxística y 95\% tenía bajo riesgo de sangrado según puntaje de HAS-BLED. Los factores de riesgo cardiovascular más frecuentes fueron: hipertensión arterial en $100 \%$, dislipidemia en $50 \%$ y diabetes mellitus tipo 2 en $25 \%$. Con respecto a los parámetros ecocardiográficos, se encontró una dilatación moderada de la aurícula izquierda con un diámetro de 45,9 \pm $6,8 \mathrm{~mm}$ y un volumen de $27,0 \pm 4,6 \mathrm{~cm}^{2}$, la fracción de eyección del ventrículo izquierdo se encontraba conservada, promedio de 58,8 $\pm 7,9 \%$.

Los efectos de rivaroxaban sobre FXa, TAT, GT y TP, en diferentes tiempos: basal, a las 3 y 24 $\mathrm{h}$ posteriores a la primera dosis de rivaroxaban y a los 30 días de tratamiento se muestran en la Tabla 2. En la Figura 1 se muestran los cambios en los niveles plasmáticos de rivaroxaban a las 3 y $24 \mathrm{~h}$ (previo a la siguiente dosis del fármaco) y a los 30 días $(\mathrm{p}<0,0001) \mathrm{y}$, asimismo, la variación de la actividad anti-FXa $(\mathrm{p}=0,012)$. Se observó una correlación significativa y persistente entre la actividad anti-FXa y los niveles plasmáticos del rivaroxaban, a las $3 \mathrm{~h}$ y $24 \mathrm{~h}$ y a los 30 días de tratamiento $(r=0,61, p 0,008 ; r=0,85, p<0,00001$ y $r=0,99, p<0,00001$, respectivamente).

En el análisis de la GT en plasma pobre en plaquetas (Figura 2), se observa que hubo una disminución significativa de la velocidad $(\mathrm{p}<0,0026)$, peak ( $\mathrm{p}<0,0001)$ y GT global $(\mathrm{p}<0,0001)$, la que se correlacionó con la actividad anti-FXa con una disminución de la generación de trombina global a las $24 \mathrm{~h}(\mathrm{r}=-0.57 ; \mathrm{p}=0,016)$ y a los 30 días $(r=-0,74 ; p=0,0038)$; al contrario, la GT global en plasma rico en plaquetas (Figura 3 ) no 
Tabla 1. Características de los pacientes

\begin{tabular}{|c|c|c|}
\hline & Variables & Promedios - frecuencias \\
\hline \multirow{7}{*}{$\begin{array}{l}\text { Características } \\
\text { basales }\end{array}$} & Sexo femenino & $60,0 \%$ \\
\hline & Edad (años) & $76,3 \pm 8,0$ \\
\hline & Índice de masa corporal (kg/m²) & $27,3 \pm 4,8$ \\
\hline & Clearence creatinina $\left(\mathrm{mL} / \mathrm{min} / 1,73 \mathrm{~m}^{2}\right)^{*}$ & $65,5 \pm 27,8$ \\
\hline & Fibrilación auricular paroxística & $85,0 \%$ \\
\hline & Puntaje $\mathrm{CHA}_{2} \mathrm{DS}_{2}-\mathrm{VASC} \geq 2$ puntos & $100 \%$ \\
\hline & Puntaje HAS-BLED $<3$ puntos & $95 \%$ \\
\hline \multirow{6}{*}{$\begin{array}{l}\text { Factores de riesgo } \\
\text { cardiovascular }\end{array}$} & Hipertensión arterial & $100 \%$ \\
\hline & Dislipidemia** & $50,0 \%$ \\
\hline & Diabetes mellitus tipo 2 & $25,0 \%$ \\
\hline & Tabaquismo crónico activo & $20,0 \%$ \\
\hline & Insuficiencia cardiaca etapa B & $10,0 \%$ \\
\hline & Antecedente de accidente cerebro vascular & $5,0 \%$ \\
\hline \multirow{5}{*}{$\begin{array}{l}\text { Fármacos de acción } \\
\text { cardiovascular }\end{array}$} & IECA o ARA-II $\left.\right|^{* * *}$ & $65 \%$ \\
\hline & Estatinas & $50 \%$ \\
\hline & Beta bloqueadores & $50 \%$ \\
\hline & Amiodarona & $40 \%$ \\
\hline & Diuréticos & $40 \%$ \\
\hline \multirow{4}{*}{$\begin{array}{l}\text { Parámetros } \\
\text { ecocardiográficos }\end{array}$} & Diámetro aurícula izquierda (mm) & $45,9 \pm 6,8$ \\
\hline & Volumen aurícula izquierda $\left(\mathrm{cm}^{2}\right)$ & $27,0 \pm 4,6$ \\
\hline & Fracción de eyección del ventrículo izquierdo (\%) & $58,8 \pm 7,9$ \\
\hline & Presión sistólica de arteria pulmonar (mm de Hg) & $37,0 \pm 12,3$ \\
\hline
\end{tabular}

*Clearence de creatininemia estimado según fórmula de Crockcroft y Gault. **Antecedente de dislipidemia obtenido de registro clínico. ${ }^{* *}$ IECA: inhibidores de la enzima convertidora de angiotensina; ARA-II: antagonista de los receptores de angiotensina II.

Tabla 2. Correlación de la actividad anti-FXa, con los niveles plasmáticos de Rivaroxaban y factores procoagulantes

\begin{tabular}{|c|c|c|c|c|}
\hline & \multirow[b]{2}{*}{ Basal } & \multicolumn{3}{|c|}{ Post-administración de Rivaroxaban } \\
\hline & & $3 \mathbf{h}$ & $24 \mathrm{~h}$ & 30 días \\
\hline Actividad anti-FXa (UI/mL) & 0 & $4,8 \pm 2,5$ & $0,52 \pm 0,51$ & $0,62 \pm 0,4$ \\
\hline NP₹ Rivaroxaban (ng/mL) & 0 & $\begin{array}{c}221,6 \pm 128 \\
(r=0,61 ; p=0,008)\end{array}$ & $\begin{array}{c}22,1 \pm 21,3 \\
(r=0,85 ; p<0,0001)\end{array}$ & $\begin{array}{c}23,2 \pm 17,4 \\
(r=0,99 ; p<0,0001)\end{array}$ \\
\hline GT en PPP执 (nM) & $99,9 \pm 38,2$ & $\begin{array}{c}3,7 \pm 8,9 \\
(r=-0,18 ; p=0,48)\end{array}$ & $\begin{array}{c}43,7 \pm 57,1 \\
(r=-0,57 ; p=0,016)\end{array}$ & $\begin{array}{c}37,2 \pm 54,2 \\
(r=-0,74 ; p=0,0038)\end{array}$ \\
\hline GT en PRP $¥ \neq \ddagger(n M)$ & $232,6 \pm 76$ & $\begin{array}{c}187,2 \pm 87,2 \\
(r=-0,03 ; p=0,99)\end{array}$ & $\begin{array}{c}243,8 \pm 105,6 \\
(r=-0,17 ; p=0,05)\end{array}$ & $\begin{array}{c}257,4 \pm 107,9 \\
(r=0,019 ; p=0,94)\end{array}$ \\
\hline Complejos TAT (ng/mL) & $1,9 \pm 1,1$ & $\begin{array}{c}1,6 \pm 0,5 \\
(r=-0,04 ; p=0,86)\end{array}$ & $\begin{array}{c}1,6 \pm 0,5 \\
(r=0,13 ; p=0,06)\end{array}$ & $\begin{array}{c}1,6 \pm 0,6 \\
(r=0,09 ; p=0,75)\end{array}$ \\
\hline Tiempo de protromboina (\%) & $97,6 \pm 10,6$ & $\begin{array}{c}49,2 \pm 12,2 \\
(r=-0,86 ; p=0,019)\end{array}$ & $\begin{array}{c}85,0 \pm 12,0 \\
(r=-0,33 ; p=0,18)\end{array}$ & $\begin{array}{c}84,5 \pm 13,8 \\
(r=-0,63 ; p=0,02)\end{array}$ \\
\hline
\end{tabular}

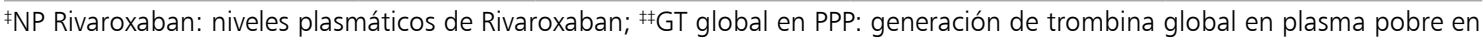
plaquetas; ${ }^{\prime \prime}$ GT global en PRP: generación de trombina global en plasma rico en plaquetas. 

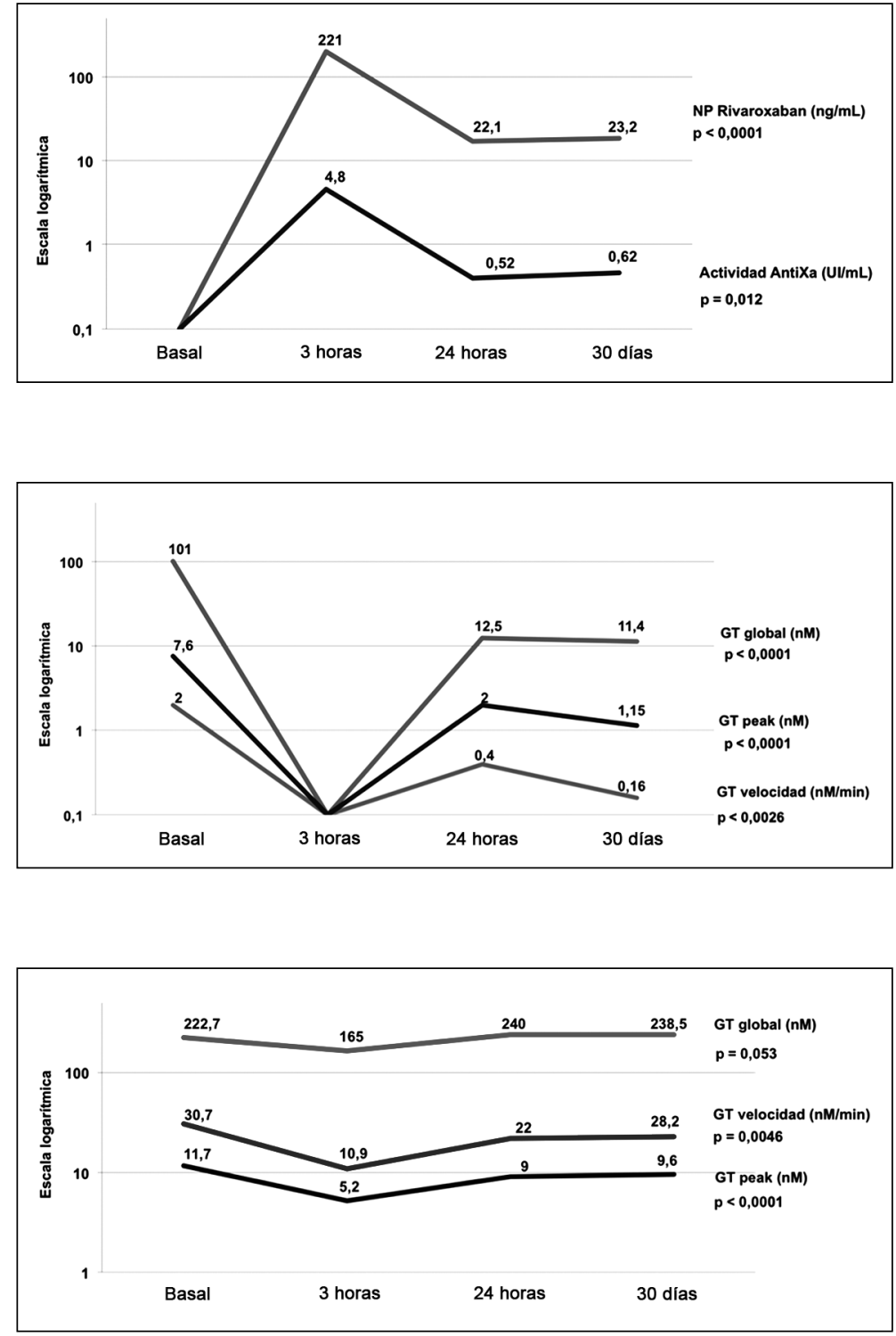

fue afectada significativamente por el fármaco $(\mathrm{p}=0,053)$. Los niveles plasmáticos basales de TAT no se modificaron con el uso de rivaroxaban $(\mathrm{p}=0,064)$.

En la Figura 4 se observa una prolongación significativa del TP basal, $49,2 \pm 12,2 \%$, con respecto al efecto máximo de rivaroxaban a las $3 \mathrm{~h}$, manteniéndose en niveles de $84,5 \pm 13,8 \%$ hasta los 30 días ( $\mathrm{p}<0,0001)$. En cuanto al TP (Figura 5), se pudo establecer una correlación entre el nivel de
Figura 2.

Figura 3.

Figura 1.

rivaroxaban y el TP a las $3 \mathrm{~h}(\mathrm{r}-0,33 ; \mathrm{p} 0,019)$ y a los 30 días de tratamiento $(\mathrm{r}-0,63 ; \mathrm{p} 0,02)$.

La dosis de inicio y mantención de rivaroxaban fue de $20 \mathrm{mg}$ diarios en 15 pacientes y de $15 \mathrm{mg}$ diarios en los 5 restantes (ajustada por edad, peso y función renal). No hubo diferencias significativas con ambas dosis sobre los parámetros evaluados.

Durante el período de seguimiento no se reportaron sangrados menores, mayores, ni eventos cardiovasculares adversos. 


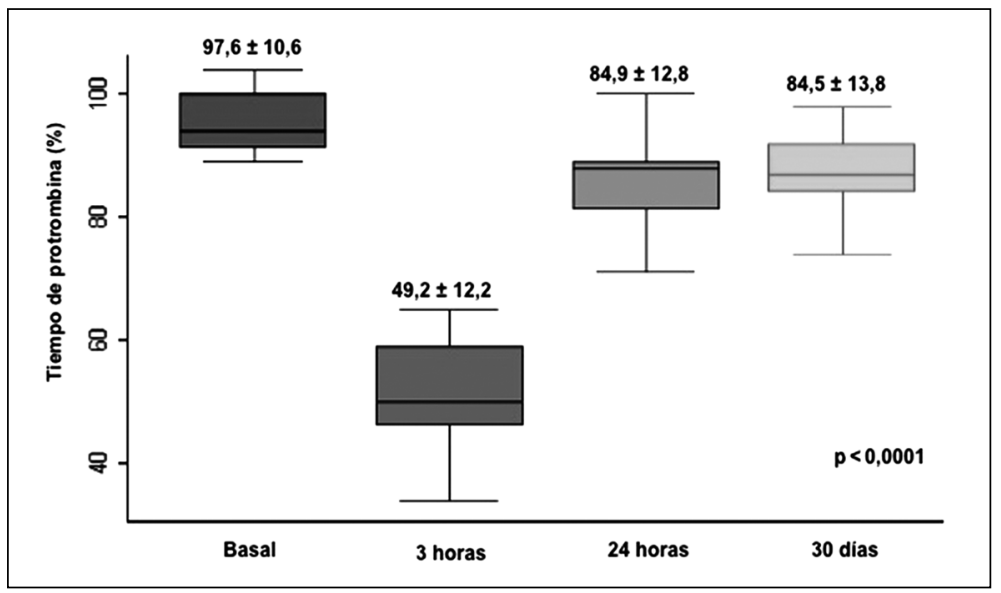

Figura 4.

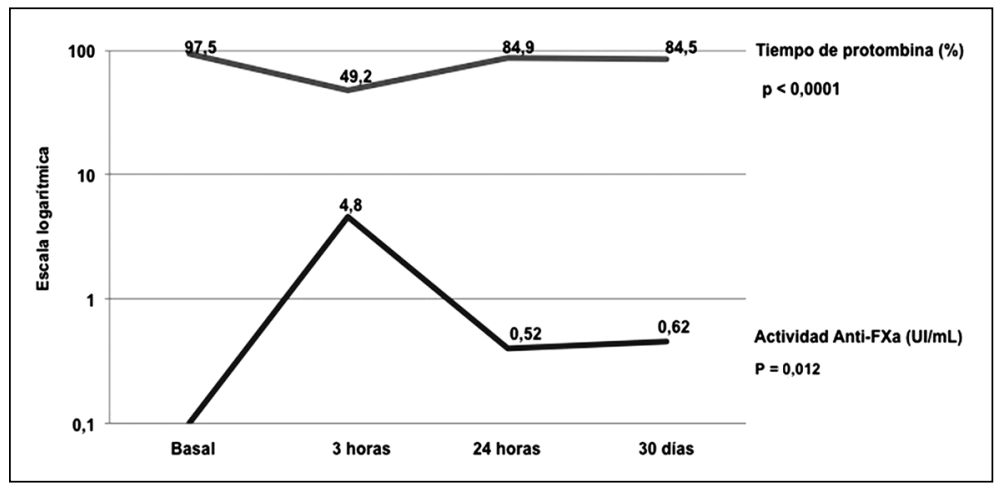

Figura 5.

\section{Discusión}

Nuestro estudio confirma un efecto anticoagulante rápido y sostenido en el tiempo de rivaroxaban en pacientes con FA no valvular de reciente comienzo y que no habían recibido tratamiento anticoagulante previo. La muestra de pacientes fue homogénea en cuanto a edad, promedio de 76 años y un perfil de riesgo moderado de tromboembolismo sistémico de acuerdo al puntaje de $\mathrm{CHA}_{2} \mathrm{DS}_{2}$-VASc.

Como se ha reportado en estudios in vitro y en sujetos sanos los niveles plasmáticos de rivaroxaban alcanzan su valor máximo entre las 2 y $4 \mathrm{~h}$ de administrada la droga. Nuestro estudio confirma la persistencia de niveles plasmáticos dentro de un rango terapéutico a las $24 \mathrm{~h}$ de administración de la droga y al cabo de 30 días de tratamiento, lo cual implica un nivel de confiabilidad respecto de la recomendación de administrar el medicamento sólo en una dosis diaria, a diferencia de otros anticoagulantes directos que teniendo una vida media similar deben administrarse en dos dosis diarias. La administración de una dosis única asegura una mejor adhesión al tratamiento a largo plazo ${ }^{4,9,10,18}$.

El rivaroxaban inhibe competitivamente, in vitro, el FXa (Ki de $0,4 \mathrm{nM}$ ), con una selectividad 10.000 veces superior en comparación con las proteínas relacionadas en la cascada de la coagulación, a través de este mecanismo rivaroxaban bloquea la formación de trombina ${ }^{15}$. En nuestro estudio encontramos una reducción rápida y significativa de la generación de trombina medida en plasma pobre en plaquetas, la que se correlacionó con la disminución de la actividad anti-FXa del fármaco. Si bien la actividad anti Xa máxima de rivaroxaban alcanza su mayor efecto a las $3 \mathrm{~h}$, esta persiste a las $24 \mathrm{~h}$, tanto en la primera evaluación como en 
la efectuada a los 30 días. Los niveles observados de actividad anti Xa son similares a los obtenidos con heparinas de bajo peso molecular.

El efecto de rivaroxaban sobre la GT en plasma rico en plaquetas fue significativo a las $3 \mathrm{~h}$, aunque de mucho menor magnitud que en plasma pobre en plaquetas y afectando solamente su valor máximo y la velocidad para generar trombina. Es probable que la generación de una superficie procoagulante sobre las plaquetas activadas y expresión de factor tisular, sobrepasa la capacidad de rivaroxaban de inhibir localmente la generación de trombina. Estos resultados están de acuerdo a lo reportado por Perzbron y cols., que sugieren, incluso, que la adición de un inhibidor de la activación de las plaquetas podría incrementar la capacidad antitrombótica de la droga, lo cual es un elemento a considerar en pacientes que han requerido implante reciente de stents por síndrome coronario agudo y tienen además $\mathrm{FA}^{16,17}$.

A diferencia de los inhibidores indirectos del $\mathrm{FXa}$, rivaroxaban no precisa de antitrombina para alcanzar su efecto e inhibe tanto el FXa libre como el unido a la fibrina, así como el FXa en el complejo de protrombinasa ${ }^{11,18}$, lo que se corrobora con la ausencia de efecto de la droga sobre niveles de TAT encontrado en nuestro estudio. Al mismo tiempo, confirmamos que el tiempo de protrombina se alarga cuando se alcanzan los valores plasmáticos máximos de la droga para retornar gradualmente a la normalidad a las $24 \mathrm{~h}$. Si bien el tiempo de protrombina no se utiliza para evaluar el efecto anticoagulante de rivaroxaban, su determinación en algunas condiciones clínicas puede ser útil para estimar indirectamente el tiempo transcurrido en relación a la última dosis s,19-21 $^{9}$.

La FA confiere un estado protrombótico o de hipercoagulabilidad, con alteraciones de la hemostasia, trombosis, activación de plaquetas y disfunción endotelial ${ }^{22}$. Se ha asociado a niveles elevados de marcadores procoagulantes, tales como GT y complejos TAT $^{23-24}$, los que persisten elevados incluso en pacientes que están en tratamiento anticoagulante oral ${ }^{25}$. Los niveles plasmáticos elevados de fragmentos $1+2$ de protrombina y los complejos TAT, reflejan la GT in vivo y sus niveles han sido asociados a mayor riesgo de accidente cerebrovascular ${ }^{26-27}$. Es probable que en pacientes con FA y un perfil de riesgo más alto, los niveles de factores procoagulantes se encuentren más elevados, como ha sido descrito en varias publicaciones previas $^{28-30}$. De todos modos, existe un consenso de la necesidad de iniciar terapia anticoagulante oral en pacientes que tiene un puntaje de riesgo de $\mathrm{CHA}_{2} \mathrm{DS}_{2}$-VASc igual o mayor a 2, como fue el caso de nuestra serie ${ }^{31-32}$.

La anticoagulación oral con inhibidores de la vitamina $\mathrm{K}$ se asocia a una reducción significativa de embolías sistémicas, pero tiene limitaciones por la dificultad en su dosificación y en lograr niveles terapéuticos estables. La introducción de nuevos anticoagulantes orles, inhibidores directos del FXa o de la trombina, permiten alcanzar un efecto terapéutico más estable y seguro, pero su mayor limitación es la dificultad para medir el nivel de anticoagulación en un momento dado. Los resultados de nuestro estudio confirman que este fármaco alcanza un efecto anticoagulante rápido y sostenido en el tiempo a través de la inhibición del FXa y de la GT.

El estudio ROCKET AF, en pacientes con FA no valvular, demostró que rivaroxaban en dosis de $20 \mathrm{mg}$ una vez al día fue, al menos, tan eficaz como la warfarina en la prevención del accidente cerebrovascular isquémico y de embolías sistémicas ${ }^{4,33}$. Aunque en la práctica clínica habitual la monitorización de la droga no es necesaria, existen situaciones, tales como insuficiencia renal, edades extremas, sangrado agudo o necesidad de procedimiento invasivo, en que conocer el grado de anticoagulación puede ser necesario. Las posibilidades de determinar actividad del factor anti-Xa o en su defecto del tiempo protrombina, deberían ser técnicas disponibles en la medida de que el número de pacientes en tratamiento con inhibidores directos del factor anti-Xa va en aumento, de acuerdo a los resultados de nuestro estudio.

Los resultados de este estudio contribuyen a confirmar y caracterizar el efecto anticoagulante de rivaroxaban en pacientes con FA, lo que otorga seguridad en el manejo clínico de la droga.

Agradecimientos: Al personal del Centro de Investigación Clínica Universidad Católica por su dedicación y colaboración con este proyecto.

\section{Referencias}

1. Ruff CT, Giugliano RP, Braunwald E, Hoffman EB, Deenadayalu N, Ezekowitz MD, et al. Comparison of the efficacy and safety of new oral anticoagulants with war- 
farin in patients with atrial fibrillation: a meta-analysis of randomised trials. Lancet 2014; 383 (9921): 955-62.

2. Kakkar AK, Mueller I, Bassand JP, Fitzmaurice DA, Goldhaber SZ, Goto S, et al. Risk profiles and antithrombotic treatment of patients newly diagnosed with atrial fibrillation at risk of stroke: perspectives from the International, observational, prospective GARFIELD registry. PLoS One 2013; 8 (5): e63479.

3. Connolly SJ, Ezekowitz MD, Yusuf S, Eikelboom J, Oldgren J, Parekh A, et al. RE-LY Steering Committee and Investigators. Dabigatran versus Warfarin in $\mathrm{Pa}$ tients with Atrial Fibrillation. N Engl J Med 2009; 361: 1139-51.

4. Patel MR, Mahaffey KW, Garg J, Pan G, Singer DE, Hacke W, et al. The ROCKET AF Steering Committee for the ROCKET AF Investigators. Rivaroxaban versus Warfarin in Nonvalvular Atrial Fibrillation. N Engl J Med 2011; 365: 883-91.

5. Granger CB, Alexander JH, McMurray JJ, Lopes RD, Hylek EM, Hanna M, et al. The ARISTOTLE Committees and Investigators. Apixaban versus Warfarin in Patients with Atrial Fibrillation. N Engl J Med 2011; 365: 981-92.

6. Giugliano RP, Ruff CT, Braunwald E, Murphy SA, Wiviott SD, Halperin JL, et al. ENGAGE AF-TIMI 48 Investigators. Edoxaban versus Warfarin in Patients with Atrial Fibrillation. N Engl J Med 2013; 369: 2093-104.

7. Martínez-Rubio A, Martínez-Torrecilla R. Evidencias actuales de los nuevos anticoagulantes orales en el tratamiento de la fibrilación auricular no valvular: comparación de subestudios. Rev Esp Cardiol 2015; 68: 185-9; 369: 2093-104.

8. Mateo J. Nuevos anticoagulantes orales y su papel en la práctica clínica. Rev Esp Cardiol Supl 2013; 13: 33-41.

9. Francart SJ, Hawes EM, Deal AM, Adcock DM, Gosselin R, Jeanneret C, et al. Performance of coagulation tests in patients on therapeutic doses of Rivaroxaban. A cross-sectional pharmacodynamic study based on peak and trough plasma levels. Thromb Haemost 2014; 111 (6): 1133-40.

10. Gulseth MP, Michaud J, Nutescu EA. Rivaroxaban: an oral direct inhibitor of factor Xa. Am J Health Syst Pharm 2008; 65 (16): 1520-9.

11. Graff J, Von Hentig N, Misselwitz F, Kubitza D, Becka M, Breddin HK. Effects of the oral, direct factor Xa inhibitor Rivaroxaban on platelet-induced thrombin generation and prothrombinase activity. J Clin Pharmacol 2007; 47 (11): 1398-407.

12. Douxfils J, Tamigniau A, Chatelain B, Chatelain C, Wallemacq P, Dogné JM, et al. Comparison of calibrated chromogenic anti-Xa assay and PT tests with LC-MS/
MS for the therapeutic monitoring of patients treated with Rivaroxaban. Thromb Haemost 2013; 110: 723-31.

13. Quiroga T, Goycoolea M, Giesen PL, Morales M, Muñoz $\mathrm{B}$, Aranda E, et al. Thrombin generation in platelet-poor plasma is normal in patients with hereditary mucocutaneous haemorrhages. Pathophysiol Haemost Thromb 2003; 33: 30-5.

14. Hemker HC, Giesen PLA, Ramjee M, Wagen-voord R, Béguin S. The thrombogram: Monitoring thrombin generation in platelet rich plasma. Thromb Haemost 2000; 83: 589-91.

15. Tyan F, Valerie G, Sarah A. Rivaroxaban: An Oral Factor Xa Inhibitor. Clinical Therapeutics 2013; 35 (1): 4-27.

16. Perzborn E, Heitmeier S, Laux VJ. Effects of Rivaroxaban on Platelet Activation and Platelet-Coagulation Pathway Interaction: In Vitro and In Vivo Studies. Cardiovasc Pharmacol Ther 2015 Apr 6. pii: 1074248415578172.

17. Gibson CM, Mehran R, Bode C, Halperin J, Verheugt F, Wildgoose P, et al. An open-label, randomized, controlled, multicenter study exploring two treatment strategies of rivaroxaban and a dose-adjusted oral vitamin $\mathrm{k}$ antagonist treatment strategy in subjects with atrial fibrillation who undergo percutaneous coronary intervention (PIONEER AF-PCI). American Heart Journal, Volume 169, Issue 4, 472-478.e5.

18. Samama MM. The mechanism of action of Rivaroxaban -an oral, direct Factor Xa inhibitor- compared with other anticoagulants. Thromb Res 2011; 127: 497-504.

19. Harenberg J, Marx S, Weiss C, Krämer R, Samama M, Schulman S. Subcommittee on Control of Anticoagulation of the ISTH. Report of the Subcommittee of Control of Anticoagulation on the determination of the anticoagulant effects of Rivaroxaban. J Thromb Haemost 2012; 10 (7): 1433-6.

20. Tripodi A, Chantarangkul V, Guinet C, Samama MM. The International Normalized Ratio calibrated for Rivaroxaban has the potential to normalize prothrombin time results for Rivaroxaban-treated patients: results of an in vitro study. J Thromb Haemost 2011; 9 (1): 226-8.

21. Lindhoff-Last E, Ansell J, Spiro T, Samama MM. Laboratory testing of Rivaroxaban in routine clinical practice: when, how, and which assays. Ann Med 2013; 45 (5-6): 423-9.

22. Hatzinikolaou-Kotsakou E, Kartasis Z, Tziakas D, Hotidis A, Stakos D, Tsatalas K. Atrial Fibrillation and Hypercoagulability: Dependent on Clinical Factors or/ and on Genetic Alterations? Journal of Thrombosis and Thrombolysis 2003; 16 (3): 155-61.

23. Heppell RM, Berkin KE, Mc Lenachan JM, Davies JA. Haemostatic and haemodynamic abnormalities asso- 
ciated with left atrial thrombosisin non-rheumatic atrial fibrillation. Heart 1997; 77: 407-11.

24. Iga K, Izumi Ch, Inoko M, Kitaguchi S, Himura Y, Gen $\mathrm{H}$, et al. Increased thrombin-antithrombin III complex during an episode of paroxysmal atrial fibrillation. International Journal of Cardiology 1998: 66; 53-156.

25. Van Kuilenburg J, Lappegard K, Sexton J, Plesiewicz I, Lap P, Bouwels P. Persisting thrombin activity in elderly patients with atrial fibrillation on oral anticoagulation is decreased by anti-inflammatory therapy with intensive cholesterol-lowering treatment. Journal of Clinical Lipidology 2011: 5; 273-80.

26. Feinberg W, Pearce L, Hart R, Cushman, M, Cornell E, Lip G, et al. For the Stroke Prevention in Atrial Fibrillation Investigators. Markers of Thrombin and Platelet Activity in Patients With Atrial Fibrillation Correlation With Stroke Among 1531 Participants in the Stroke Prevention in Atrial Fibrillation III Study. Stroke 1999; 30: 2547-53.

27. Topcuoglu MA, Haydari D, Ozturk S, Ozcebe OI, Saribas O. Plasma levels of coagulation and fibrinolysis markers in acute ischemic stroke patients with lone atrial fibrillation. Neurol Sci 2000: 21; 235-40.

28. Pérez L, Corbalán R, Acevedo M, Pereira J, Braun S, Tapia J, et al. Hipercoagulabilidad en fibrilación auricular y su relación con factores de riesgo para embolia sistémica. Rev Med Chile 2002; 130 (10): 1087-94.

29. Lip GY, Lip PL, Zarifis J, Watson RDS, Bareford D,
Lowe GD, et al. Fibrin Ddimer and b-thromboglobulin as markers of thrombogenesis and platelet activation in atrial fibrillation. Circulation 1996; 94: 425-31.

30. Roldán V, Marín F, García A, Tello-Montoliu A, Lip GY. Is an advanced age an additive risk factor to the prothrombotic or hypercoagulable state in atrial fibrillation? International Journal of Cardiology, Volume 110, Issue 2, 265-6.

31. Camm J, Lip GY, De Caterina R, Savelieva I, Atar D, Hohnloser S, et al. ESC Committee for Practice Guidelines (CPG). 2012 focused update of the ESC Guidelines for the management of atrial fibrillation: an update of the 2010 ESC Guidelines for the management of atrial fibrillation. Developed with the special contribution of the European Heart Rhythm Association. Eur Heart J 2012; 33 (21): 2719-47.

32. January CT, Wann LS, Alpert JS, Calkins H, Cigarroa JE, Cleveland JC, et al. American College of Cardiology/ American Heart Association Task Force on Practice Guidelines. 2014 AHA/ACC/HRS guideline for the Management of patients with atrial fibrillation: a report of the American College of Cardiology/American Heart Association Task Force on Practice Guidelines and the Heart Rhythm Society. J Am Coll Cardiol 2014; 2, 64 (21): 2305-7.

33. López-Sendón J, Merino J. Resultados e implicaciones del estudio ROCKET. Rev Esp Cardiol Supl 2013; 13: 42-6. 